\title{
Primary Appendix Lymphoma: Case Report and Review of the Literature
}

\author{
Burak Ardicli *, [MD] \\ Ayse Karaman ${ }^{1},[\mathrm{MD}]$ \\ Sule Yesil ${ }^{2}$, [MD] \\ Nilufer $\mathrm{Arda}^{3},[\mathrm{MD}]$ \\ Gurses Sahin², [MD] \\ Ismet Faruk Ozguner ${ }^{1}$, [MD] \\ 1, Dr. Sami Ulus Maternity and Children's Training and \\ Research Hospital, Department of Pediatric Surgery. \\ 2, Dr. Sami Ulus Maternity and Children's Training and \\ Research Hospital, Department of Pediatric Oncology \\ 3, Dr. Sami Ulus Maternity And Children's Training And \\ Research Hospital, Department of Pediatric Surgery \\ and Pathology \\ *Corresponding Author: Burak Ardicli, M.D. \\ Dr. Sami Ulus Maternity and Children's Training and \\ Research Hospital \\ 06080, Ankara/TURKEY \\ Phone: +90 3123056208 \\ DOI: https://doi.org/10.32552/2018.ActaMedica.306 \\ e-mail: burakardicli@gmail.com
}

\section{ree ABSTRACT Cen}

Appendectomy is the most frequent emergent surgical procedure in childhood. Carcinomas are the most frequent neoplasms in the appendix, other neoplasms such as carcinoid tumors and lymphomas may see less frequently. Primary tumors of the appendix are very rare. We report a patient presented with acute appendicitis diagnosed with B-cell lymphoma after pathological examination.

Keywords: Childhood lymphoma, B-cell lymphoma, appendix lymphoma

Received: 22 June 2018, Accepted: 18 September 2018,

Published online: 30 September 2018

\section{INTRODUCTION}

Appendectomy is the most frequent emergency surgical procedure in childhood [1]. Pathological examination of the appendectomy specimens usually demonstrate diagnosis of acute appendicitis [2]. Appendiceal neoplasms may present with appendicitis [3]. Carcinomas are the most frequent neoplasms in the appendix, other neoplasms such as carcinoid tumors and lymphomas may seen less frequently [2]. Primary tumors of the appendix are very rare [4]. We report a patient presented with acute appendicitis is diagnosed with lymphoma after pathological observation. This is a rare form of non-Hodgkin's (NHL) lymphoma.

\section{Literature review}

PubMed Central (US National Library of Medicine, 8600 Rockville Pike, Bethesda, Maryland, USA) and Google Scholar (Google Inc., 1600 Amphitheatre Parkway, Mountain View, California, USA) searches plus references of these articles revealed cases of appendicitis, lymphoma.

All cases were reviewed and patient details (age, sex, pathologic diagnosis, clinical appendicitis, surgical methods, and accompanying diseases) were noted, SPSS 16.0 was used for statistical analysis (mean value, standart deviation, minimum and maximum value). 


\section{CASE REPORT}

An 11-year-old boy was admitted to our clinic with the complaint of abdominal pain and nausea. Intermittent abdominal pain, which was aggravated in last day, was learned from the history. Physical examination revealed right lower quadrant tenderness and guarding. The patient had skin rash secondary to psoriasis on his lower extremities. In laboratory assessment, white blood cell count and C-reactive protein levels were $7900 / \mu \mathrm{L}(5000-11800 \mu \mathrm{L})$ and $23.5 \mathrm{mg} / \mathrm{L}(0-8 \mathrm{mg} / \mathrm{L})$ respectively. Blood biochemistry parameters were in normal range. Abdominal $X$-ray demonstrated air-fluid levels in the right

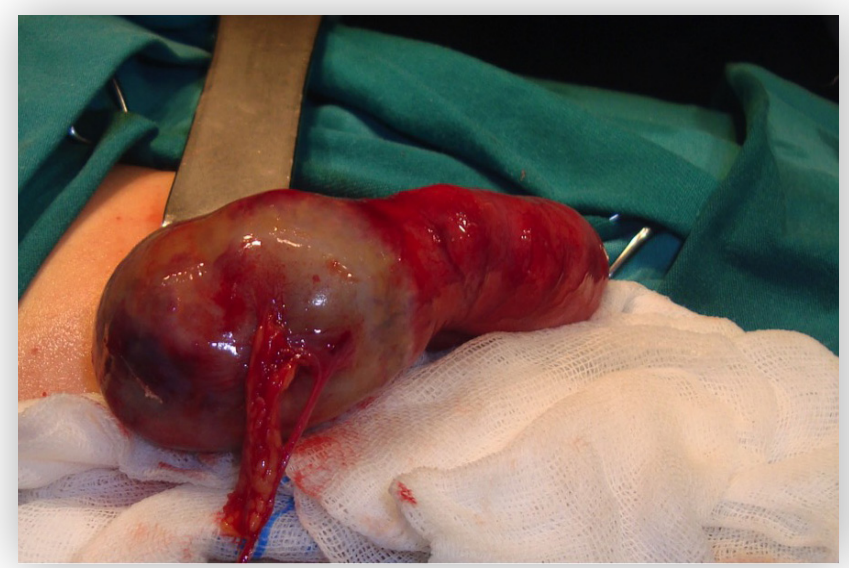

Figure 1. The Appendix was widely thickened.

\section{Literature Review}

There have been 169 cases of appendiceal lymphoma reported until 2015 (Table 1). These cases there were 76 adults, 34 children, and in 59 cases age group wasn't reported (Figure 3). Male to female ratio was 52/36, and in 81 cases sex wasn't reported. Mean age of adults was $47.03+19.15(18-84)$, mean age of children was $9.15+$ 4.699 (3-17), and in 86 cases age wasn't reported.

Sixty-one (36\%) patients presented with right lower quadrant pain and underwent appendectomy. Other sypmtoms were abdominal mass, nausea, vomiting and weight loss.

B-cell lymphoma was the most common pathologic diagnosis (78\%), followed by T-cell lymphoma (5\%) and Hodgkin's disease (2\%) respectively. The classification lower quadrant and scoliosis. The patient was operated with the initial diagnosis of acute appendicitis. There were plenty of serous fluid in peritoneal cavity. The appendix and cecum were widely thickened (Figure 1). Appendectomy was performed. Postoperative serum level of LDH 324 IU/L (115-257 IU/L) was slightly higher than normal. Peripheral blood smear and bone marrow smear were normal. Postoperative course was uneventful. Oral feeding was started on the postoperative day. Pathological examination of appendix revealed centroblastic subtype of diffuse large B-cell lymphoma (Figure 2). The patient was discharged on the fifth postoperative day and he is on chemotherapy in the pediatric oncology department.

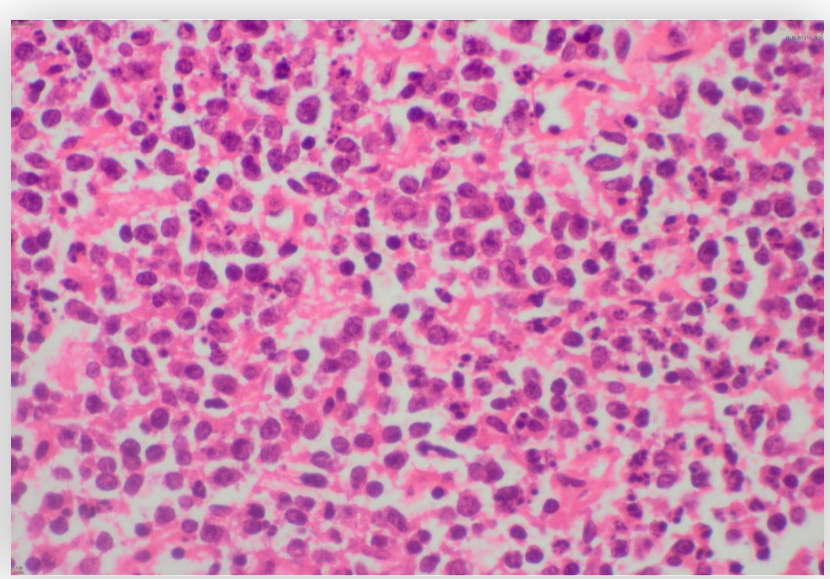

Figure 2. Diffuse large B-cell lymphoma. Centroblastic variant. The tumour cells have a polymorphic and polylobated appearance.

was unspecified in $15 \%$ of cases and in 3 cases pathological diagnosis wasn't reported (Figure 4).

Appendectomy was the most common surgical method $(81 \%)$, followed by right hemicolectomy (12\%) and ileocecal resection (7\%), respectively.and in sixty-three cases surgical methods were not reported (Figure 5).

Accompanying diseases was reported disseminated primary intestinal lymphoma [1], Acquired Immune Deficiency Syndrome [1], Primary nasal NK/T cell lymphoma [1], Crohn's Disease [1], posttransplant lymphoprolipherative disorder [1], renal transplant [1], and chronic lymphocytic leukemia/Small lymphocytic lymphoma [1] (Table 1)

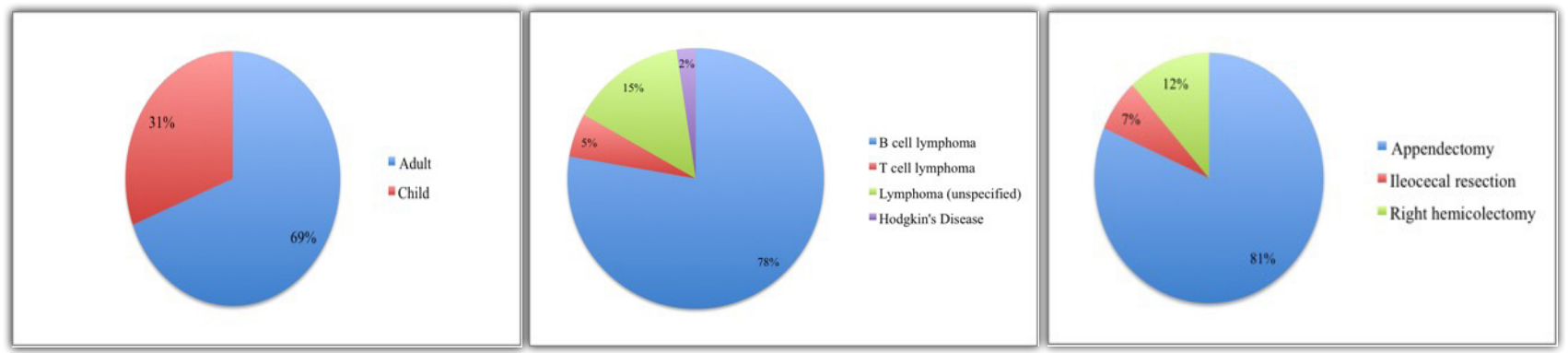

Figure 3. Age group according to apendiceal lymphoma
Figure 4. Pathologic distribution according Figure 5. Surgical methods to appendiceto appendiceal lymphoma al lymphoma 
Table 1. Appendiceal lymphoma clinical data in the literature

\begin{tabular}{|c|c|c|c|c|c|c|c|c|c|}
\hline & Age & $\begin{array}{l}\text { Gende } \\
\mathrm{r}\end{array}$ & $\begin{array}{l}\text { Age } \\
\text { group }\end{array}$ & $\begin{array}{l}\text { Number } \\
\text { of cases }\end{array}$ & Pathologic findings & $\begin{array}{l}\text { Clinical } \\
\text { Appendiciti } \\
\text { s }\end{array}$ & Operation & Survey & $\begin{array}{l}\text { Accompan } \\
\text { ying } \\
\text { diseases }\end{array}$ \\
\hline Warren, 1898 (11) & & M & & 1 & Lymphoblastic sarcoma & & Ileocaecectomy & $>4$ years & \\
\hline Davis, 1900 (12) & 51 & M & Adult & 1 & Lymphoblastic sarcoma & & Appendectomy & $>5$ months & \\
\hline Paterson, 1903 (11) & 39 & M & Adult & 1 & Lymphoblastic sarcoma & & Appendectomy & $\begin{array}{c}\text { Postoperative } \\
\text { died }\end{array}$ & \\
\hline Bernays, 1905 (11) & 29 & $\mathrm{~F}$ & Adult & 1 & Lymphoblastic sarcoma & & Appendectomy & 9 months, died & \\
\hline DeJong, 1907 (11) & & M & & 1 & Lymphoblastic sarcoma & & Appendectomy & & \\
\hline $\begin{array}{l}\text { Carwardine, } 1907 \\
\text { (13) }\end{array}$ & 45 & $\mathrm{~F}$ & Adult & 1 & Lymphoblastic sarcoma & & Appendectomy & & \\
\hline Wilhelm, 1919 (11) & 17 & M & Child & 1 & Lymphoma & & & & \\
\hline Powers, 1911 (11) & 12 & $\mathrm{~F}$ & Child & 1 & Lymphoblastic sarcoma & & Appendectomy & 10 weeks, died & \\
\hline Wright, 1911 (14) & 17 & M & Child & 1 & Lymphoblastic sarcoma & & $\begin{array}{c}\text { Right } \\
\text { hemicolectomy }\end{array}$ & $>3$ years & \\
\hline White, 1913 (11) & 25 & $\mathrm{~F}$ & Adult & 1 & Lymphoblastic sarcoma & & Appendectomy & $>4$ years & \\
\hline $\begin{array}{l}\text { Rohdenburg, } 1919 \\
\text { (11) }\end{array}$ & 4 & M & Child & 1 & Lymphoblastic sarcoma & & Caecectomy & $>10$ months & \\
\hline Goldstein, 1921 (11) & 25 & $\mathrm{~F}$ & Adult & 1 & Lymphoblastic sarcoma & & Appendectomy & & \\
\hline Lehman, 1925 (11) & 20 & $\mathrm{~F}$ & Adult & 1 & & & & & \\
\hline Friend, 1926 (11) & 9 & $\mathrm{~F}$ & Child & 1 & Lymphoblastic sarcoma & & Appendectomy & $\begin{array}{c}\text { Postoperative } \\
\text { died }\end{array}$ & \\
\hline Capecchi, 1927 (11) & 8 & M & Child & 1 & Lymphoblastic sarcoma & & Appendectomy & & \\
\hline Stout, 1925 (11) & $\begin{array}{l}8 \\
9\end{array}$ & $\mathrm{~F}$ & Child & 2 & Giant follicular lymphoma & & & $\begin{array}{c}\text { Postoperative } \\
\text { died }\end{array}$ & \\
\hline Evans, 1932 (15) & 55 & M & Adult & 1 & Giant follicular lymphoma & 1 & Appendectomy & $>3$ years & \\
\hline Ullman, 1932 (11) & & & & 2 & Giant follicular lymphoma & & Appendectomy & & \\
\hline Bizard, 1938 (11) & 27 & M & Adult & 1 & Lymphoblastic sarcoma & & Appendectomy & $>3.5$ years & \\
\hline Ruggieri, 1938 (11) & 39 & M & Adult & 1 & Giant follicular lymphoma & & & & \\
\hline Knox, 1945 (16) & 4 & M & Child & 1 & Lymphoblastic sarcoma & & Appendectomy & $>15$ months & \\
\hline Morhead, 1945 (11) & $\begin{array}{l}12 \\
26 \\
33\end{array}$ & $\begin{array}{l}\mathrm{M} \\
\mathrm{M} \\
\mathrm{F}\end{array}$ & $\begin{array}{l}\text { Child } \\
\text { Adult } \\
\text { Adult }\end{array}$ & 3 & $\begin{array}{l}\text { Giant follicular lymphoma } \\
\text { Giant follicular lymphoma } \\
\text { Giant follicular lymphoma }\end{array}$ & & $\begin{array}{l}\text { Appendectomy } \\
\text { Appendectomy } \\
\text { Appendectomy }\end{array}$ & & \\
\hline McSwain, 1945 (11) & $\begin{array}{l}39 \\
37\end{array}$ & F & $\begin{array}{l}\text { Adult } \\
\text { Adult }\end{array}$ & 2 & $\begin{array}{c}\text { Giant follicular lymphoma } \\
\text { Lymphosarcoma }\end{array}$ & & $\begin{array}{l}\text { Appendectomy } \\
\text { Appendectomy }\end{array}$ & $\begin{array}{l}>2 \text { years } \\
>4 \text { years }\end{array}$ & \\
\hline Galloway, 1949 (17) & & & & 1 & Giant follicular lymphoma & & Othopsy & Died & \\
\hline Jason, 1949 (11) & 34 & $\mathrm{~F}$ & Adult & 1 & Giant follicular lymphoma & & Appendectomy & & \\
\hline Clarke, 1950 (11) & 32 & M & Adult & 1 & Lymphosarcoma & & No resection & 1 month, died & \\
\hline Henley, 1954 (11) & 38 & M & Adult & 1 & Diffuse large cell lymphoma & & Appendectomy & $>3$ years & \\
\hline Rosenberg ,1961 (6) & & & - & 13 & Lymphosarcoma & 1 & & & \\
\hline
\end{tabular}




\begin{tabular}{|c|c|c|c|c|c|c|c|c|c|}
\hline Collins, 1963 (6) & & & - & 11 & Lymphosarcoma & & & & \\
\hline Dorfman, 1965 (6) & & & - & 3 & Burkitt's lymphoma & & & & \\
\hline Glick, 1966 (11) & 4 & M & Child & 1 & Diffuse large cell lymphoma & & $\begin{array}{c}\text { Right } \\
\text { hemicolectomy }\end{array}$ & $>12$ years & \\
\hline Jenkin, 1969 (18) & & & Child & 8 & Lymphoma & 6 & & & \\
\hline Loehr, 1969 (19) & & $\mathrm{F}$ & Adult & 2 & Lymphoma & & Appendectomy & $>9$ years & \\
\hline Lewin, 1978 (20) & 15 & & Child & 1 & Lymphoma & 1 & Appendectomy & $>4$ years & \\
\hline $\begin{array}{l}\text { Contreary, } 1980 \\
\text { (21) }\end{array}$ & & & Adult & 3 & $\begin{array}{l}\text { Giant follicular Lymphoma } \\
\qquad(1) ; 2 x ?\end{array}$ & & & & \\
\hline Franchini, 1979 (6) & 25 & $\mathrm{~F}$ & Adult & 1 & Stem cell & & & & \\
\hline Nanji, 1983 (22) & 22 & M & Adult & 1 & $\begin{array}{c}\text { Undifferentiated Burkitt's } \\
\text { type }\end{array}$ & 1 & Appendectomy & & \\
\hline $\begin{array}{l}\text { Schmutzer, } 1975 \\
\text { (23) }\end{array}$ & & & Adult & 3 & Lymphoma & & Appendectomy & & \\
\hline Sin, $1980(24)$ & $\begin{array}{l}8 \\
10\end{array}$ & $\begin{array}{l}M \\
M\end{array}$ & $\begin{array}{l}\text { Child } \\
\text { Child }\end{array}$ & 2 & Burkitt's lymphoma & & Appendectomy & $\begin{array}{c}>3 \text { years } \\
>18 \text { months }\end{array}$ & \\
\hline Saitou, 1981 (11) & 20 & $\mathrm{~F}$ & Adult & 1 & Lymphoma & & $\begin{array}{l}\text { Right } \\
\text { hemicolectomy }\end{array}$ & 7 months, died & \\
\hline $\begin{array}{l}\text { Murakuni, } 1982 \\
\text { (11) }\end{array}$ & & & & 1 & Diffuse large cell lymphoma & & $\begin{array}{c}\text { Right } \\
\text { hemicolectomy }\end{array}$ & & \\
\hline Ghani, 1984 (6) & & M & Adult & 1 & Burkitt's lymphoma & 1 & & & \\
\hline Swerdlow, 1984 (6) & 34 & $\mathrm{~F}$ & Adult & 1 & Caribbean T cell lymphoma & & & & \\
\hline Mori, 1985 (7) & 70 & $\mathrm{~F}$ & Adult & 1 & $\begin{array}{c}\text { Lymphoma, small } \\
\text { lymphocytic }\end{array}$ & & $\begin{array}{l}\text { Ileocecal resection } \\
\text { with mesenteric } \\
\text { lymph node } \\
\text { dissection }\end{array}$ & $>3$ years & \\
\hline Back, 1986 (25) & 57 & M & Adult & 1 & $\begin{array}{l}\text { Unclassified high grade } \\
\text { lymphoma }\end{array}$ & 1 & Appendectomy & $>40$ months & \\
\hline Stewart, 1986 (26) & 33 & $\mathrm{~F}$ & Adult & 1 & B cell lymphoma & 1 & Appendectomy & $>18$ months & \\
\hline Chan, 1987 (27) & & & & 2 & Lymphoma & 2 & Appendectomy & & $\begin{array}{c}\text { Dissemine } \\
\text { primary } \\
\text { intestinal } \\
\text { lymphoma }\end{array}$ \\
\hline Chawla, 1990 (6) & 34 & $\mathrm{~F}$ & Adult & 1 & $\begin{array}{l}\text { Poorly differentiated } \\
\text { lymphocytic lymphoma }\end{array}$ & & & & \\
\hline Shimada, 1990 (28) & 48 & M & Adult & 1 & $\begin{array}{l}\text { Diffuse large cell lymphoma, } \\
\text { B cell type }\end{array}$ & & Ileocecal resection & & \\
\hline $\begin{array}{l}\text { Carpenter, } 1991 \\
\text { (29) }\end{array}$ & 65 & M & Adult & 1 & $\begin{array}{l}\text { Diffuse infiltration of small } \\
\text { cell lymphoma }\end{array}$ & & $\begin{array}{c}\text { Right } \\
\text { hemicolectomy } \\
\text { with paraaortic } \\
\text { lymph node } \\
\text { resection }\end{array}$ & & \\
\hline Caine, 1990 (6) & 3 & $\mathrm{~F}$ & Child & 1 & Burkitt's lymphoma & 1 & & & \\
\hline
\end{tabular}




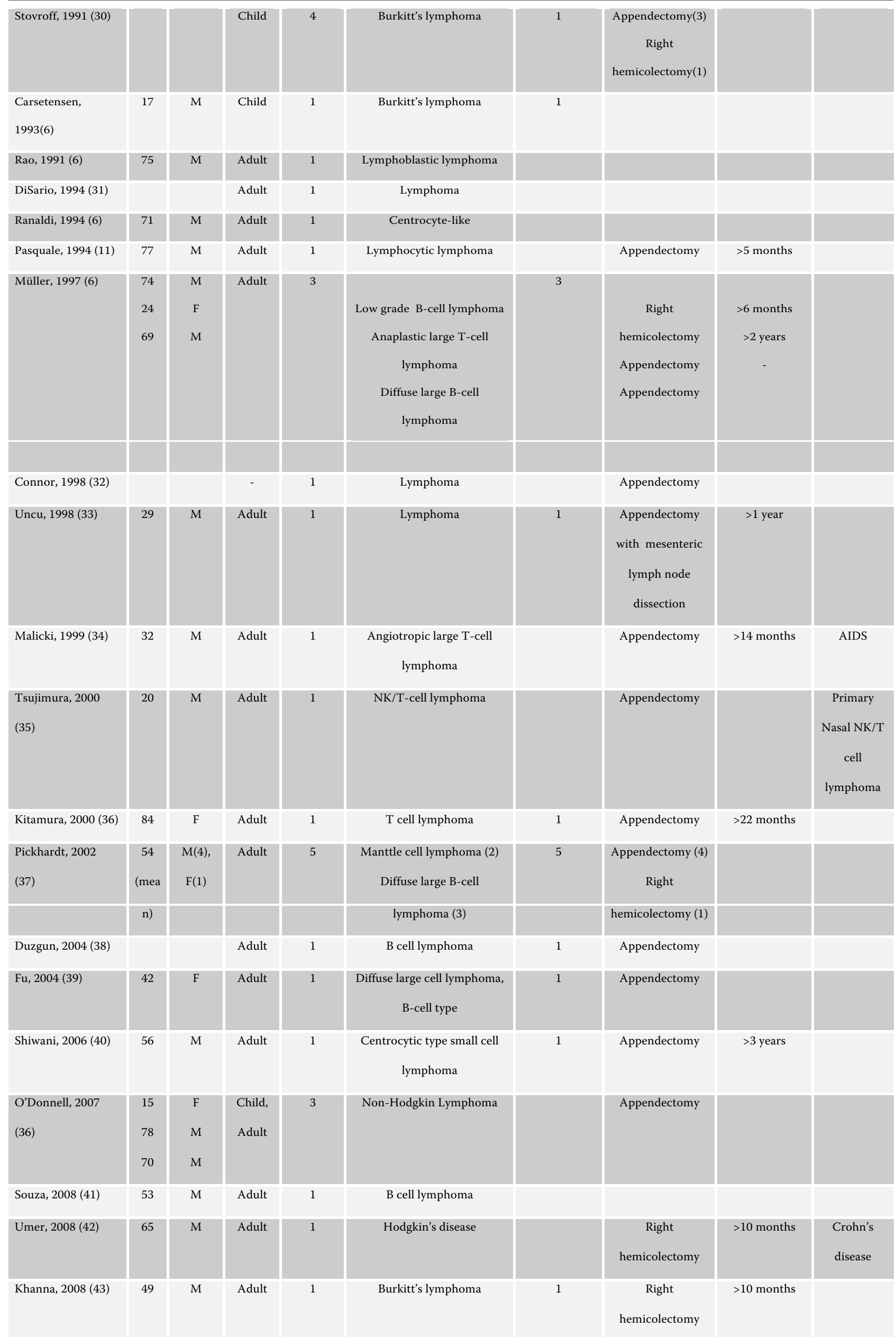




\begin{tabular}{|c|c|c|c|c|c|c|c|c|c|}
\hline Medlicott, 2008 (44) & 53 & $\mathrm{~F}$ & Adult & 1 & $\begin{array}{l}\text { Diffuse large cell lymphoma, } \\
\text { B-cell type }\end{array}$ & 1 & Appendectomy & & $\begin{array}{c}\text { Posttranspla } \\
\text { nt } \\
\text { lymphoprolif } \\
\text { erative } \\
\text { disorder }\end{array}$ \\
\hline Radha, 2008 (45) & 61 & M & Adult & 1 & B-cell lymphoma & & Ileocecal resection & & \\
\hline Marte, 2008 (46) & 6 & F & Child & 1 & $\begin{array}{c}\text { Mucosa-associated lymphoid } \\
\text { tissue lymphoma }\end{array}$ & 1 & Appendectomy & $>15$ months & \\
\hline $\begin{array}{l}\text { Gustafsson, } 2008 \\
\text { (4) }\end{array}$ & & & - & 12 & $\begin{array}{l}\text { Diffuse large cell lymphoma, } \\
\text { B-cell type (11), Hodgkin's } \\
\text { disease (1) }\end{array}$ & & & & \\
\hline $\begin{array}{l}\text { Toyomasu, } 2009 \\
(47)\end{array}$ & 74 & $\mathrm{~F}$ & Adult & 1 & $\begin{array}{c}\text { Mucosa-associated lymphoid } \\
\text { tissue lymphoma }\end{array}$ & 1 & Ileocecal resection & & \\
\hline Weine, 2009 (48) & 76 & M & Adult & 1 & Hodgkin's Disease & 1 & Appendectomy & & \\
\hline \multirow[t]{2}{*}{ Ghasmei, 2010 (49) } & 22 & M & Adult & 1 & Diffuse large cell lymphoma, & 1 & Appendectomy & $>2$ years & \\
\hline & & & & & B-cell type & & & & \\
\hline Miyazaki, 2010 (50) & 60 & $\mathrm{~F}$ & Adult & 1 & $\begin{array}{c}\text { Mucosa-associated lymphoid } \\
\text { tissue lymphoma }\end{array}$ & 1 & $\begin{array}{l}\text { Right } \\
\text { hemicolectomy } \\
\text { with partial } \\
\text { resection of the } \\
\text { right ureter }\end{array}$ & $>2$ years & \\
\hline Abdalla, 2010 (51) & 49 & M & Adult & 1 & Burkitt's lymphoma & 1 & Appendectomy & $>1$ month & \\
\hline Bhardwaj, 2010 (52) & 14 & M & Child & 1 & Burkitt's lymphoma & 1 & $\begin{array}{c}\text { Right } \\
\text { hemicolectomy }\end{array}$ & & \\
\hline Baek, 2011 (53) & 37 & M & Adult & 1 & $\begin{array}{l}\text { Diffuse large cell lymphoma, } \\
\text { B-cell type }\end{array}$ & 1 & Appendectomy & & \\
\hline Akbulut, 2011 (54) & & & $\begin{array}{l}\text { Child, } \\
\text { Adult }\end{array}$ & 14 & $\begin{array}{l}\text { B-cell lymphoma (12) } \\
\text { T-cell lymphoma (2) }\end{array}$ & 14 & Appendectomy & & \\
\hline Ratuapli, 2011 (55) & 45 & M & Adult & 1 & Large T cell lymphoma & 1 & Appendectomy & & $\begin{array}{l}\text { Renal } \\
\text { transplant }\end{array}$ \\
\hline Quigley, 2012 (56) & 64 & $\mathrm{~F}$ & Adult & 1 & $\begin{array}{l}\text { Small lymphocytic lymphoma } \\
\text { and Hodgkin transformation }\end{array}$ & 1 & Appendectomy & & $\begin{array}{l}\text { Chronic } \\
\text { lymphocytic } \\
\text { leukemia / } \\
\text { Small } \\
\text { lymphocytic } \\
\text { lymphoma }\end{array}$ \\
\hline Linden, 2012 (2) & 71 & M & Adult & 1 & Mantle cell lymphoma & 1 & Appendectomy & & \\
\hline $\begin{array}{l}\text { Matsushita, } \\
\text { 2013(57) }\end{array}$ & 7 & M & Child & 1 & T-cell lymphoma & 1 & Appendectomy & $>2$ years & \\
\hline Yilmaz, 2013 (58) & & & Adult & 1 & B-cell lymphoma & & Appendectomy & & \\
\hline Weledji, 2014 (59) & $\begin{array}{l}13 \\
18\end{array}$ & F & $\begin{array}{l}\text { Child, } \\
\text { Adult }\end{array}$ & 2 & Burkitt's lymphoma & 2 & $\begin{array}{l}\text { Appendectomy } \\
\text { Ileocecal resection }\end{array}$ & $\begin{array}{l}>8 \text { years } \\
>9 \text { years }\end{array}$ & \\
\hline Sun, 2014 (60) & & & Adult & 1 & T-cell lymphoma & & & & \\
\hline
\end{tabular}




\begin{tabular}{|c|c|c|c|c|c|c|c|}
\hline Ghosal, 2014 (61) & 3 & & Child & 1 & Hodgkin's disease & 1 & $\begin{array}{l}\text { Abdominal lymph } \\
\text { node biopsy }\end{array}$ \\
\hline Guo, 2015 (62) & 43 & $\mathrm{~F}$ & Adult & 1 & $\begin{array}{c}\text { Diffuse large cell lymphoma, } \\
\text { B-cell type }\end{array}$ & 1 & Appendectomy \\
\hline Chae, 2015 (63) & 75 & $M$ & Adult & 1 & Mantle cell lymphoma & 1 & Appendectomy \\
\hline
\end{tabular}

\section{DISCUSSION}

Lymphoma often causes gastrointestinal involvement. Clinical presentation shows great variation. Diarrhea, weight loss, and intussusception are the most common signs and symptoms [5]. Lymphomatous infiltration of the appendix and lymphoma associated with acute appendicitis is very rare. Primary appendicular lymphoma is diagnosed earlier than other gastrointestinal lymphomas as it gives rise symptoms earlier in the disease and an early stage [6]. Abdominal X-ray is generally nonspecific. Ultrasonography reveals abdominal mass, enlarged lymph nodes, and ascites. Abdominal computerized tomography scanning should be performed with oral and intravenous contrast agent. Thus, a characteristic appearance of contrast-filled loops of bowel, trapped and encased by large soft tissue masses is seen. 'Omental cakes' sign is shown by CT. However, abdominal X-ray and US imaging are preferred more often than CT in the setting of acute abdominal pain and other gastrointestinal symptoms [5]. Computed tomography is preferred less often because of the risk of radiation. Therefore, preoperative diagnosis is rare. Mori et al. described correct diagnosis in 19 of 39 cases. Many of these patients presented with acute abdomen [7].

Gastrointestinal system lymphomas can occur four parts; lymphoid tissue, the lamina propria, intraepithelial lymphocytes, and mesenteric lymph nodes [4]. The incidence of small intestine and colon lymphomas is 0.22 to 0.35 and 0.1 to 0.21 , respectively. Lymphomas of the appendix were rare showing $1.7 \%$ of all appendix tumors [4]. Small intestine lymphomas are frequently of diffuse large B-cell type. Burkitt lymphoma is a rare. It frequently occurs in the ileo-cecal region. Appendiceal $B$ cell lymphoma was analyzed Burkitt's lymphoma rate $\% 13$ in this study. The most common presentation of ileo-cecal region lymphoma is abdominal pain. On the other hand, according to this study, only $36 \%$ of cases presented with classical clinical signs and symtoms of appendicitis. Appendicceal lymphoma rarely presents with palpable abdominal mass [8].

Colon lymphoma include $10 \%$ of all lymphomas and Generally occur in the cecum [9]. Colonic lymphomas are frequently of diffuse large B-cell type. The most common presentations are abdominal pain. Colon lymphoma is usually seen in the elderly and associated with inflammatory bowel disease, HIV/ AIDS and immunosuppressive treatment [10]. In this study, which examined the appendix lymphoma cases, there was only one case of AIDS and three cases were on immunosuppresive agents. Also, our patient had psoriasis. Psoriasis is a chronic inflammatory disease and it might be at increased risk of cancer due to chronic inflammation and immunosuppressive drugs. A study that is systematic literature review showed that the risk of non-Hodgkin lymphoma appears to be slightly increased in psoriasis.

The T-cell lymphoma is extremely rare. It is associated with immune compromised conditions. The gastrointestinal tract is the most common site for extranodal involvement of non-Hodgkin's lymphoma; appendiceal lymphoma is exceedingly rare [11]. Hodgkin's diseases, was found $2 \%$ in appendix lymphomas (Figure 4).

In literature, the most common surgical method is appendectomy (Figure 5). On the other hand, extensive excision may be needed. What should be the surgical method for appendiceal malignancies? Our recommendation is not to make a wide excision without a pathological diagnosis.

\section{CONFLICT OF INTEREST}

The authors declare that there are no conflicts of interest in this study. 
[1] Addiss DG, Shaffer N, Fowler BS, et al. The epidemiology of appendicitis and appendectomy in the United States. Am J Epidemiol 1990; 132(5): 910-25.

[2] Linden M, Gopal A, Edlefsen K. Acute appendicitis in a man undergoing therapy for mantle cell lymphoma. Case Rep Hematol 2012; 2012: 868151.

[3] Ruoff C, Hanna L, Zhi W, et al. Cancers of the appendix: review of the literatures. ISRN Oncol 2011; 2011: 728579.

[4] Gustafsson BI, Siddique L, Chan A, et al. Uncommon cancers of the small intestine, appendix and colon: an analysis of SEER 1973-2004, and current diagnosis and therapy. Int J Oncol 2008; 33(6): 1121-31.

[5] Carachi R, Azmy AAF, Grosfeld JL. The surgery of childhood tumours. 2nd ed. ed. Berlin; London: Springer; 2008.

[6] Muller G, Dargent JL, Duwel V, et al. Leukaemia and lymphoma of the appendix presenting as acute appendicitis or acute abdomen. Four case reports with a review of the literature. J Cancer Res Clin Oncol 1997; 123(10): 560-4.

[7] Mori M, Kusunoki T, Kikuchi M, et al. Primary malignant lymphoma of the appendix. Jpn J Surg 1985; 15(3): 230-3.

[8] Yin L, Chen CQ, Peng CH, et al. Primary small-bowel non-Hodgkin's lymphoma: a study of clinical features, pathology, management and prognosis. J Int Med Res 2007; 35(3): 406-15.

[9] Romaguera J, Hagemeister FB. Lymphoma of the colon. Curr Opin Gastroenterol 2005; 21(1): 80-4.

[10] Wong MT, Eu KW. Primary colorectal lymphomas. Colorectal disease 2006; 8(7): 586-91.

[11] Pasquale MD, Shabahang M, Bitterman $P$, et al. Primary lymphoma of the appendix. Case report and review of the literature. Surg Oncol 1994; 3(4): 243-8.

[12] Davis TG. Sarcoma of vermiform appendix. JAMA 1900; XXXV(24): 1556-7.

[13] Carwardine T. Primary Sarcoma of the Vermiform Appendix. BMJ 1907; 2(2451): 1771

[14] Wright G. Primary Sarcoma of the Vermiform Appendix. BMJ 1911; 2(2638): 150-1.

[15] Evans A. Lymphoma of the Vermiform Appendix. Proc R Soc Med 1932; 25(3): 332-3.

[16] Knox G. Lymphosarcoma primary in the appendix. Arch Surgery 1945; 50(6): 288-92.

[17] Galloway WH, Owens EJ. Primary lymphosarcoma of appendix occurring in childhood. BMJ 1949; 2(4641): 1387, illust.

[18] Jenkin RD, Sonley MJ, Stephens CA, et al. Primary gastrointestinal tract lymphoma in childhood. Radiology 1969; 92(4): 763-7.

[19] Loehr WJ, Mujahed Z, Zahn FD, et al. Primary lymphoma of the gastrointestinal tract: a review of 100 cases. Ann Surg 1969; 170(2): 232-8.

[20] Lewin KJ, Ranchod M, Dorfman RF. Lymphomas of the gastrointestinal tract: a study of 117 cases presenting with gastrointestinal disease. Cancer 1978; 42(2): 693-707.

[21] Contreary K, Nance FC, Becker WF. Primary lymphoma of the gastrointestinal tract. Ann Surg 1980; 191(5): 593-8.

[22] Nanji AA, Anderson FH. Burkitt's lymphoma with acute appendicitis. Arch Surg 1983; 118(11): 1352.

[23] Schmutzer KJ, Bayar M, Zaki AE, et al. Tumors of the appendix. Dis Colon Rectum 1975; 18(4): 324-31.

[24] Sin IC, Ling ET, Prentice RS. Burkitt's lymphoma of the appendix: report of two cases. Hum Pathol 1980; 11(5): 465-70.
[25] Back H, Gustavsson B, Ridell B, et al. Primary gastrointestinal lymphoma incidence, clinical presentation, and surgical approach. J Surg Oncol 1986; 33(4): 234-8.

[26] Stewart RJ, Mirakhur M. Primary malignant lymphoma of the appendix. Ulster Med J 1986; 55(2): 187-9.

[27] Chan W, Fu KH. Value of routine histopathological examination of appendices in Hong Kong. J Clin Pathol 1987; 40(4): 429-33.

[28] Shimada K, Sato M, Haruyama S, et al. [A primary malignant lymphoma of the appendix]. Gan No Rinsho 1990; 36(11): 2086-91.

[29] Carpenter BW. Lymphoma of the appendix. Gastrointest Radiol 1991; 16(3): 256-8.

[30] Stovroff MC, Coran AG, Hutchinson RJ. The role of surgery in American Burkitt's lymphoma in children. J Pediat Surg 1991; 26(10): 1235-8.

[31] DiSario JA, Burt RW, Kendrick ML, et al. Colorectal cancers of rare histologic types compared with adenocarcinomas. Dis Colon Rectum 1994; 37(12): 1277-80.

[32] Connor SJ, Hanna GB, Frizelle FA. Appendiceal tumors: retrospective clinicopathologic analysis of appendiceal tumors from 7,970 appendectomies. Dis Colon Rectum 1998; 41(1): 75-80.

[33] Uncu H, Erdem E, Tuzuner A. Primary malignant lymphoma of the appendix (a case report and review of the literature). Acta Chir Hung 1998; 37(1-2): 11-6.

[34] Malicki DM, Suh YK, Fuller GN, et al. Angiotropic (intravascular) large cell lymphoma of T-cell phenotype presenting as acute appendicitis in a patient with acquired immunodeficiency syndrome. Arch Pathol Lab Med 1999; 123(4): 335-7.

[35] Tsujimura H, Takagi T, Tamaru J, et al. Involvement of the appendix in a relapsed case of primary nasal NK/T-cell lymphoma. Leuk Lymphoma 2000; 37(5-6): 633-4.

[36] O'Donnell ME, Badger SA, Beattie GC, et al. Malignant neoplasms of the appendix. Int J Colorectal Dis 2007; 22(10): 1239-48.

[37] Pickhardt PJ, Levy AD, Rohrmann CA, Jr., et al. Non-Hodgkin's lymphoma of the appendix: clinical and CT findings with pathologic correlation. AJR 2002; 178(5): 1123-7.

[38] Duzgun AP, Moran M, Uzun S, et al. Unusual findings in appendectomy specimens: Evaluation of 2458 cases and review of the literature. Indian J Surg 2004; 66: 221-6.

[39] Fu TY, Wang JS, Tseng HH. Primary appendiceal lymphoma presenting as perforated acute appendicitis. JCMA 2004; 67(12): 629-32.

[40] Shiwani MH. Primary malignant lymphoma of the appendix associated with acute appendicitis. JCPSP 2006; 16(1): 79-80.

[41] Souza FF, Israel DA. PET/CT appearance of non-Hodgkin lymphoma of the vermiform appendix. Clin Nucl Med 2008; 33(10): 694-6.

[42] Umer MA, Date RS, Mellor S, et al. Hodgkin's disease of appendix: report of a case. Colorectal Dis 2009; 11(9): 985-7.

[43] Khanna M, Buddhavarapu SR. Primary Burkitt's Lymphoma Of The Appendix Presenting As Acute Abdomen: A Case Report. J Radiol Case Rep 2008; 2(5): 9-14.

[44] Medlicott SA, Cox H, Dupre M, et al. Systemic illnesses unexpectedly presenting as acute appendicitis: case studies. Can J Surg 2008; 51(1): 73-4.

[45] Radha S, Afroz T, Satyanarayana G. Primary marginal zone B-cell lymphoma of appendix. Indian J Pathol Microbiol 2008; 
51(3): 392-4.

[46] Marte A, Sabatino MD, Cautiero P, et al. Unexpected finding of laparoscopic appendectomy: appendix MALT lymphoma in children. Pediatr Surg Int 2008; 24(4): 471-3.

[47] Toyomasu Y, Tsutsumi S, Yamaguchi S, et al. Laparoscopyassisted ileocecal resection for mucosa-associated lymphoid tissue lymphoma of the appendix: case report. Hepatogastroenterology 2009; 56(93): 1078-81.

[48] Weine DM, Gelfand RM, Fraser CR, et al. The first reported case of nodular lymphocyte-predominant Hodgkin's lymphoma of the appendix. Am J Gastroenterol 2009; 104(7): 1860-1.

[49] Ghasmei M, Abedian-Kenari S. A Primary Diffuse Large B-Cell Lymphoma of Appendix. Iran Red Crescent Med J 2010; 12(5): 576-8.

[50] Miyazaki T, Ishiguro T, Ishibashi K, et al. Mucosa-associated lymphoid tissue lymphoma of the appendix vermiformis. Int Surg 2010; 95(1): 27-32.

[51] Abdalla MF, El-Hennawy HM. Unusual presentation for primary appendiceal lymphoma: A case report. Indian J Surg 2010; 72(Suppl 1): 289-92.

[52] Bhardwaj N, Bains SK, Ortonowski G, et al. A case of Burkitt's lymphoma presenting as suspected acute appendicitis. Afr J Paediatr Surg 2010; 7(3): 214-5.

[53] Baek JM, Lee JH, Sung GY, et al. Primary appendiceal lymphoma presenting as acute appendicitis. Am Surg 2011; 77(5): E87-8.

[54] Akbulut S, Tas M, Sogutcu N, et al. Unusual histopathological findings in appendectomy specimens: a retrospective analysis and literature review. World J Gastroenterol 2011; 17(15): 1961-70.
[55] Ratuapli SK, Murarka S, Miller KA, et al. Epstein Barr Viruspositive large T-cell lymphoma presenting as acute appendicitis 17 years after cadaveric renal transplant: a case report. J Med Case Rep 2011; 5: 5.

[56] Quigley B, Peker D, Zhang L. A composite small lymphocytic lymphoma and Hodgkin transformation presenting as acute appendicitis. Ann Hematol 2012; 91(5): 801-2.

[57] Matsushita Y, Takeshita M. Paediatric T-cell lymphoma of the appendix: a case report. Diagn Pathol 2013; 8: 2.

[58] Yilmaz M, Akbulut S, Kutluturk K, et al. Unusual histopathological findings in appendectomy specimens from patients with suspected acute appendicitis. World J Gastroenterol 2013; 19(25): 4015-22.

[59] Weledji EP, Ngowe MN, Abba JS. Burkitt's lymphoma masquerading as appendicitis--two case reports and review of the literature. World J Surg oncol 2014; 12: 187.

[60] Sun ZH, Zhou HM, Song GX, et al. Intestinal T-cell lymphomas: a retrospective analysis of 68 cases in China. World J Gastroenterol 2014; 20(1): 296-302.

[61] Ghosal A, Acharyya S. A rare presentation of Hodgkin's lymphoma in a very young child, with involvement of the appendix. BMJ Case Rep 2014; 2014.

[62] Guo J, Wu G, Chen X, Li X. Primary appendiceal lymphoma presenting as suspected perforated acute appendicitis: clinical, sonography and CT findings with pathologic correlation. Int J Clin Exp Pathol 2014; 7(10): 7068-71.

[63] Chae M, Kumar S, Cheema M. Mantle cell lymphoma presenting as acute appendicitis. Int J Surg Case Rep 2015; 6C: 33-5. 